\title{
Analysis on ultimate bearing capacity of the prestressed concrete beam end of dispersion anchorage under local pressure
}

\author{
YIN Xinsheng ${ }^{1, a}$, LIU Yang ${ }^{1, b^{*}}$ \\ ${ }^{1}$ Green Building Technology Engineering Center , Jilin Jianzhu University , Changchun130118, \\ China \\ ayinxinsheng06@eyou.com, ${ }^{b} 692844325 @ q q . c o m$.
}

Keywords: prestress, local pressure of edge, dispersion anchorage, ultimate bearing capacity Abstract. In this paper, a finite element software ABAQUS is carried out to calculate the ultimate load bearing capacity of the concrete under the restraint of prestressed bar. According to the failure criterion of Mohr-Coulomb, the calculation method of ultimate bearing capacity is put forward, and get the ultimate bearing capacity of concrete constrained by prestress in the beam through calculating. The analysis shows that the stress state of the concrete can be changed by prestress and the ultimate bearing capacity of the beam is improved. By comparing the calculation results, it is concluded that the theoretical formula of the failure criterion of Mohr-Coulomb is similar to the actual results, which can accurately calculate the ultimate bearing capacity of beam end.

\section{Introduction}

Partial pressure is the most common form of civil engineering, especially in the prestressed concrete structures.Domestic and foreign scholars have carried on the system research in the bearing capacity of the reinforced concrete under local pressure. And the theory of "wedge splitting" is put forward for the local pressure. From the results of the research, the edge and corner of the pressure are the most unfavorable form of partial pressure of concrete, and the ultimate bearing capacity is small. In order to improve the bearing capacity, the authors put forward a method to improve the bearing capacity by prestressing horizontal constraint.

The limit analysis of the prestressed concrete under edge local pressure

In the analysis, the concrete is assumed to be rigid plastic material, which is in accordance with the Mohr-Colomb failure criterion.

\section{Using the stress $\sigma-\tau$ on failure surface to express plastic flow deformation}

The destruction of the porous edge pressure is the failure of shear sliding. Its failure criterion and plastic flow deformation Fig. 1 Mohr-Coulomb failure criterion are shown in Figure 1.

Failure criterion:

$$
F=\tau-c+\sigma \tan \phi
$$

According to the flow rule of plastic mechanics: 
$\varepsilon=\lambda \frac{\partial F}{\partial \sigma}=\lambda \tan \phi \quad \gamma=\lambda \frac{\partial F}{\partial \tau}=\lambda$

The total deformation of the failure surface is:

$v=\sqrt{\varepsilon^{2}+\gamma^{2}}=\lambda \sqrt{1+\tan ^{2} \phi}$

$\tan \alpha=\frac{\varepsilon}{\gamma}=\tan \phi$, from that we can get $\alpha=\phi$, which shows that the total deformation in the

angle of the failure surface is $\phi$, see Figure 2.

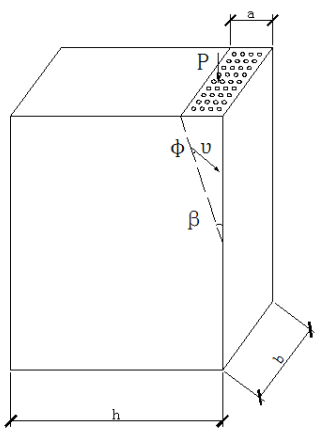

Fig. 2 Schematic diagram of component failure surface

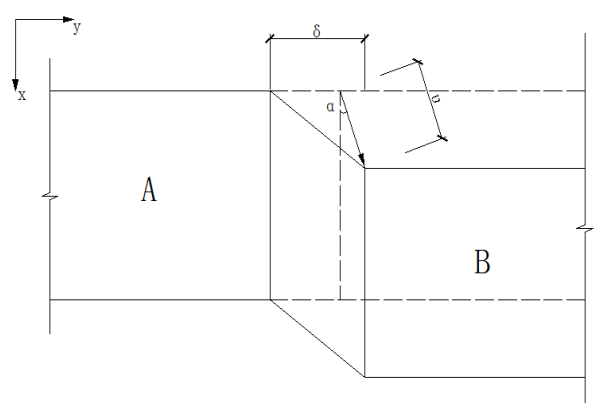

Fig. 3 Schematic diagram of sliding failure surface

\section{Internal force work}

From the basic assumption that the concrete material is rigid plastic body. Only in the failure surface plastic deformation (plastic domain) happens, see Figure 3.

The internal force produced by the concrete is:

$$
D_{c}=\sigma_{1} \varepsilon_{1}+\sigma_{2} \varepsilon_{2}+\sigma_{3} \varepsilon_{3}
$$

If the rigid body $\mathrm{A}$ is fixed, the angle between the axis " $\mathrm{X}$ " and the relative displacement " $v$ " of the rigid body B is “ $\alpha$ ” $(\phi)$. And then we can obtain:

$$
\varepsilon_{x}=0 \quad \varepsilon_{y}=\frac{v}{\delta} \sin \alpha \quad \gamma_{x y}=\frac{v}{\delta} \cos \alpha
$$

Expression of principal strain:

$$
\left\{\begin{array}{l}
\varepsilon_{1} \\
\varepsilon_{2}
\end{array}\right\}=\frac{1}{2} \cdot \frac{v \sin \alpha}{\delta} \pm \frac{1}{2} \sqrt{\frac{v^{2} \sin ^{2} \alpha}{\delta^{2}}+\frac{v^{2} \cos ^{2} \alpha}{\delta^{2}}}=\frac{1}{2} \cdot \frac{v}{\delta}(\sin \alpha \pm 1)
$$

Putting the formula (6) into the formula (4), then we will get the internal force works on the unit length along the failure surface are:

$$
\begin{aligned}
W_{I} & =\left[\sigma_{1} \frac{v}{2 \delta}(1+\sin \alpha)+\sigma_{3} \frac{v}{2 \delta}(\sin \alpha-1)\right] \delta b \\
& =\sigma_{1} \frac{v b}{2}(1+\sin \alpha)-\sigma_{3} \frac{v b}{2}(1-\sin \alpha)
\end{aligned}
$$

(Note: $b$-the width of the component) 
Failure criteria of Mohr-Coulomb concrete expressed by $\sigma_{1}$ and $\sigma_{3}$ :

$$
\begin{gathered}
K \sigma_{1}-\sigma_{3}=f_{c} \\
K=\frac{1+\sin \phi}{1-\sin \phi}
\end{gathered}
$$

We can see from the formula (9):

$1+\sin \Phi=K(1-\sin \phi)$

$$
W_{I}=\frac{1}{2} v b(1-\sin \phi) f_{c}
$$

Internal force work of prestressed reinforcement:

$W_{p}=\sigma_{p e} \cdot A_{p} v \sin \alpha$

\section{The stress bearing capacity of the side pressure under the prestressing force}

In the above analysis, the internal work of the failure surface is obtained. In limit analysis, the upper bound method of plastic mechanics is often used, which is to obtain the ultimate load by making the external force work equal to internal force work.

External force work produced by load:

$$
W_{E}=P v \cos (\phi+\beta)
$$

From internal force work is equal to external force work, we will obtain:

$P v \cos (\phi+\beta)=\frac{1}{2} v \cdot(1-\sin \phi) \cdot f_{c} \cdot \frac{b a-\pi r^{2} \cdot n}{\sin \beta}+\sigma_{p e} A_{p} v \sin \phi$

And then $P=\frac{f_{c}}{2} \cdot \frac{1-\sin \phi}{\cos (\phi+\beta)} \cdot \frac{b a-\pi r^{2} \cdot n}{\sin \beta}+\sigma_{p e} A_{p} \cdot \frac{\sin \phi}{\cos (\phi+\beta)}$

Making $\phi=30^{\circ}$, and then $\beta=45^{\circ}-\frac{\phi}{2}=45^{\circ}-\frac{30^{\circ}}{2}=30^{\circ}$

\section{The ultimate bearing capacity when the three row holes layout on the end of beam}

The model of the beam end which 3 row holes layout is shown in Figure 4. The load width is $a=160 \mathrm{~mm}$ and the length is $b=400 \mathrm{~mm}$. Concrete strength grade is C40, so $f_{c}=19.1 \mathrm{~N} / \mathrm{mm}^{2}$. There are nine holes in each row and the diameter of each hole is $18 \mathrm{~mm}$. The holes are linked up from the top of the beam end to the bottom. The diameter of prestressed bar is $18 \mathrm{~mm}$. Ultimate tensile strength of prestressed bar is $f_{p t k}=1960 \mathrm{MPa}$, and its tension control stress is $\sigma_{p e}=0.7 f_{p t k}=1372 \mathrm{MPa}$. Through the above theoretical assumption, in the case of the load width is $160 \mathrm{~mm}$, the angle between the crack surface and the vertical direction of the beam end is $30^{\circ}$. In the upper surface of the vertical down the $160 \times \sqrt{3}=277.13 \mathrm{~mm}$ range is the most effective to place prestressed bar. So place four along the width direction of the beam end with $80 \mathrm{~mm}$ spacing. Along the height direction, 4 rows, each row spacing is $50 \mathrm{~mm}$, the top row is $50 \mathrm{~mm}$ far from the top surface. Putting the above data 
and the formula (15) into the formula (14) and then the beam end ultimate bearing capacity of the three row hole layout is:

$P=\frac{19.1}{2} \times \frac{1-\sin 30^{\circ}}{\cos 60^{\circ}} \times \frac{400 \times 160-27 \times \pi \times 9^{2}}{\sin 30^{\circ}}+1372 \times \pi \times 3.5^{2} \times 16 \times \frac{\sin 30^{\circ}}{\cos 60^{\circ}}=1632.062 \mathrm{kN}$

The ultimate bearing capacity when the four row holes layout on the end of beam

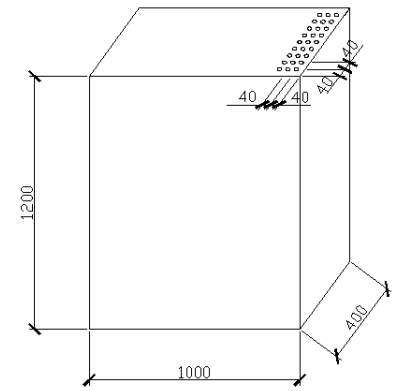

Fig. 4 Dimension sketch map of model 1

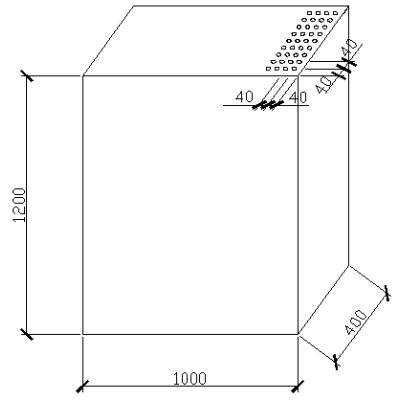

Fig. 5 Dimension sketch map of model 2

The model of the beam end which 4 row holes layout is shown in Figure 5. The load width is $a=200 \mathrm{~mm}$. Other conditions are consistent with the three row hole layout. Finally the beam end ultimate bearing capacity of the four row hole layout is:

$$
P=\frac{19.1}{2} \times \frac{1-\sin 30^{\circ}}{\cos 60^{\circ}} \times \frac{400 \times 200-36 \times \pi \times 9^{2}}{\sin 30^{\circ}}+1372 \times \pi \times 3.5^{2} \times 16 \times \frac{\sin 30^{\circ}}{\cos 60^{\circ}}=1672.571 \mathrm{kN}
$$

\section{Nonlinear finite element analysis of dispersion anchorage}

Using ABAQUS finite element software to analyze the bearing capacity of the beam end under edge local pressure. A separate unit model is established by means of the unit and the concrete element. The concrete model is introduced into the concrete model by using the elastic plastic fracture and damage model. The model uses external related hardening in order to better simulate the concrete compressive elastic plastic behavior. The bar is used alone by element, and the section area, gap, direction of the bar are defined.

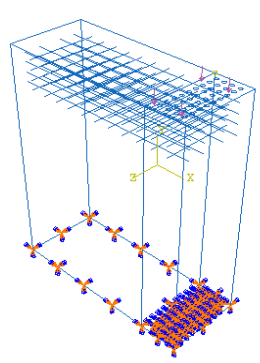

Fig. 6 Finite element analysis model

The loading area and boundary conditions of the component are shown in Figure 6. Uniform load of $50 \mathrm{MPa}$ is put on the opening hole area. The bottom of the beam is completely fixed. The prestress is realized by cooling method. The results are shown in Table 1.

Table 1 Statistical table of ultimate bearing capacity of component

\begin{tabular}{|c|c|c|}
\hline & Model 1 & Model2 \\
\hline $\begin{array}{c}\text { Ultimate bearing capacity } \\
(\mathrm{kN})\end{array}$ & 1503.529 & 1763.862 \\
\hline
\end{tabular}

Through the above results, we can see that the theoretical calculation results of the model 1 and the results of the finite element calculation are different from those of the $12.9 \mathrm{t}$, and the model 2 is $9.1 \mathrm{t}$, which is close to. 


\section{Conclusion}

By comparison, it can be seen that the calculation method of the ultimate bearing capacity of the Mohr-Coulomb theory is close to that of the finite element analysis. It can be close to the true description of the failure of shear slip theory when the beam end concrete under side pressure at the time of the failure load. To calculate the ultimate bearing capacity of its failure criterion of Mohr-Coulomb.

\section{Acknowledgements}

The selection of this thesis comes from the National Natural Science Foundation of China. The

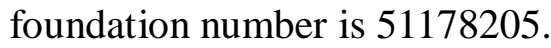

\section{References}

[1] Liu Yongyi, Guan Jianguang, Wang Chuanzhi. BEARING STRENGTH OF CONCRETE AND IT'S FAILURE MECHANISM.China Civil Engineering Journal.1985, 18 (2):53-65 